\title{
(6) OPEN ACCESS \\ A population-based incidence of acute large vessel occlusions and thrombectomy eligible patients indicates significant potential for growth of endovascular stroke therapy in the USA
}

\author{
Ansaar T Rai, ${ }^{1}$ Aaron E Seldon, ${ }^{1}$ SoHyun Boo, ${ }^{1}$ Paul S Link, ${ }^{2}$ Jennifer R Domico, ${ }^{1}$ \\ Abdul R Tarabishy, ${ }^{3}$ Noelle Lucke-Wold, ${ }^{1}$ Jeffrey S Carpenter ${ }^{1}$
}

'Department of Interventional Neuroradiology, West Virginia University, Morgantown, West Virginia, USA

${ }^{2}$ Stryker Neurovascular, Fremont, California, USA

${ }^{3}$ Department of Neuroradiology, West Virginia University, Morgantown, West Virginia, USA

Correspondence to Dr Ansaar T Rai, Interventional Neuroradiology, West Virginia University, Room 2278, HSCS, PO Box 9235, Morgantown, WV 26506, USA: ansaar.rai@gmail.com

Received 11 May 2016 Revised 21 June 2016 Accepted 24 June 2016 Published Online First 15 July 2016

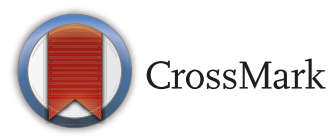

To cite: Rai AT, Seldon AE, Boo SH, et al. I Neurolntervent Surg 2017;9:722-726.

\section{ABSTRACT}

Background Data on large vessel strokes are important for resource allocation and infrastructure development.

Objective To determine an annual incidence of large vessel occlusions (LVOs) and a thrombectomy eligible patient population.

Methods All patients with acute ischemic stroke discharged over 3 years from a tertiary-level hospital serving a large geographic area were evaluated for an LVO (M1, internal carotid artery terminus, basilar artery). The incidence of LVO was determined for the hospital's 4-county primary service area (PSA, population 210000 ) based on each county's discharges and extrapolated to the US population. 'Thrombectomy eligibility' for anterior circulation LVOs was based on time (onset $<6$ hours) and imaging (Alberta Stroke Program Early CT Score (ASPECTS) $\geq 6$ ). The number of annual thrombectomy procedures was calculated for Medicare and private payer patients using federally available databases. Results 1157 patients were discharged from the hospital's PSA, of whom 129 (11.1\%, 95\% Cl 9.5\% to $13.1 \%$ ) had an LVO. This translated into an LVO incidence of 24 per 100000 people per year $(95 \% \mathrm{Cl}$ 20 to 28). 20 per 100000 people per year had anterior circulation LVOs $(95 \% \mathrm{Cl} 19$ to 22$)$, of whom $10 / 100$ 000/year ( $95 \% \mathrm{Cl} 8$ to 11) were 'thrombectomy eligible'. An additional 5/100 000/year (95\% Cl 3 to 6) presented with favorable ASPECTS after 6 hours of symptom onset. Basilar occlusion incidence was estimated at $4 / 100000 /$ year $(95 \% \mathrm{Cl} 2$ to 5$)$. These rates yield 77569 (95\% Cl 65835 to 91 091) new LVOs per year in the USA. An estimated 10284 mechanical thrombectomy procedures were performed in 2015.

Conclusions This study estimates an LVO incidence of 24 per 100000 person-years (95\% Cl 20 to 28). A current estimated annual thrombectomy rate of three procedures per 100000 people indicates significant potential increase in the volume of endovascular procedures and the need to develop systems of care.

\section{INTRODUCTION}

The current emphasis in stroke treatment is on developing systems of care which disseminate endovascular therapy. An accurate assessment of the size of the patient population who might benefit from endovascular care is an important initial step in defining the scope of the problem. The oft-quoted statistic of $\sim 795000$ acute strokes with an estimated $87 \%$ ischemic, or 691650 patients, is extrapolated from regional population studies and National Institute of Neurological Disorders and Stroke projections. ${ }^{1}$ Although important as an indicator of the total disease burden, these numbers are less relevant for determining the incidence of large vessel strokes, which are probably responsible for the bulk of morbidity, mortality, and cost associated with acute ischemic stroke (AIS). Information about this target population that has been shown to benefit from endovascular therapy is limited. ${ }^{2-5}$

This paper aimed to estimate the number of patients with stroke presenting with large vessel occlusions (LVOs). We initially determined the acute LVO rate among all patients presenting with AIS to a large tertiary level hospital in northern West Virginia, which receives almost all the patients with stroke from its primary catchment population. We used these results to estimate the rate of acute LVO for the primary population and stratified it based on time from symptom onset and favorable versus unfavorable imaging. The population-based incidence calculated from the hospital's service area was used to estimate the national incidence of LVO. The estimated rate of LVO and the current thrombectomy volume suggest the potential opportunity for endovascular stroke therapy and can serve as a marker for assessing dissemination of these services.

\section{METHODS}

Determining the incidence of large vessel strokes among all AIS discharges from the tertiary hospital

All patients discharged from hospital with a primary or secondary diagnosis of AIS based on ICD-9 diagnosis codes 433.xx (Occlusion and stenosis of precerebral arteries), 434.xx (Occlusion of cerebral arteries), and 435.xx (Transient cerebral ischemia) from an academic tertiary-level hospital in a rural setting over a 3-year period (March 2012-March 2015) were evaluated for the presence of an LVO. We used a 3-year cycle to smooth out year-to-year variations. The $\approx 700$ bed hospital has a four-county primary service area (PSA) in northern West Virginia, with a 2013 population of almost 210000 in an area of 1492 square miles. 
The total number of patients discharged from each county is reported to the West Virginia Healthcare Authority. The hospital received about $85 \%$ of patients with stroke discharged from the PSA in 2013, representing a fair estimation of the ischemic stroke burden in its catchment population. The hospital also received about $50 \%$ of patients with stroke discharged from its 11-county secondary service area (SSA), with a population of almost 213000 in an area of 5233 square miles. Additionally, the hospital receives patients with stroke from adjacent counties in Pennsylvania, Maryland, and Ohio.

A total of 2798 patients with a primary or secondary diagnosis of ischemic stroke were discharged from hospital over the 3 -year period. A baseline vascular imaging study was available in 2739 (98\%) patients, who formed the cohort for this analysis. An emergent CT angiogram was performed in 2490 $(90.9 \%)$ and an emergent MR angiogram in 249 (9.1\%) patients. An LVO was defined as middle cerebral artery main stem (M1) with or without involvement of the bifurcation branches, internal carotid artery terminus arteries (ICA-T), or basilar artery (BA). Two experienced neuroradiologists independently assessed a baseline Alberta Stroke Program Early CT Score (ASPECTS) for all patients with anterior circulation LVO on the corresponding non-contrast CT or MRI diffusionweighted images. The results were dichotomized into favorable (ASPECTS $\geq 6$ ) or unfavorable (ASPECTS <6) for intervention and any discrepant results between the two reviewers were resolved by consensus. Thus we estimated the percentage of LVOs among all discharged patients with ischemic stroke and stratified the results based on the service area, presentation time from symptom onset, and treatment eligibility based on ASPECTS

\section{Estimating the incidence of large vessel strokes among the primary service population}

As previously mentioned, total hospital discharges from each county are reported to the West Virginia Healthcare Authority (http://www.hca.wv.gov/). In 2013, the hospital received $85 \%$ of all patients with stroke from its PSA and, by accounting for the remaining $15 \%$, we estimated an annual rate of large vessel strokes per 100000 people. Using the 2015 US population of $\sim 322000$ 000, we extrapolated our results to generate rates and ranges of annual LVO incidence nationally and stratified these rates based on time of presentation and imaging.

\section{Estimating the numbers of mechanical thrombectomy cases for 2015}

The mechanical thrombectomy numbers for 2015 were estimated based on counts calculated from the Medicare Provider Analysis and Review (MedPAR) fiscal year (FY) 2015 data (FY 2017 proposed rule file) and adjusted to national counts using the 2013 National (Nationwide) Inpatient Sample (NIS) of the Agency for Healthcare Research, Quality Healthcare Cost and Utilization Project (HCUP), Rockville, Maryland, USA (http:// www.hcup-us.ahrq.gov/nisoverview.jsp). Mechanical thrombectomy cases were defined based on ischemic stroke diagnosis (ICD-9 433.xx, 434.xx, 435.xx) and ICD-9-CM procedure code 39.74 (Endovascular removal of obstruction from head and neck vessel(s)). The count of traditional Medicare discharges from MedPAR was inflated to total including Medicare Advantage Part C using an estimate of 30\% enrollment in Part C. In the 2013 NIS, Medicare represented 58.7\% of all mechanical thrombectomy cases. We estimated the total 2015 thrombectomy cases by inflating the FY2015 Medicare cases using this ratio of Medicare to private payer cases from HCUP.
The NIS is approximately a $20 \%$ stratified sample of patients discharged from US hospitals, excluding rehabilitation and longterm acute care hospitals, and contains all-payer data on hospital inpatient stays from states participating in HCUP. The hospitals are stratified based on bed size, geographical region, teaching status, urban versus rural location, and profit versus non-profit ownership. The NIS covers more than $95 \%$ of the US population, with each year including over 7 million inpatient stays. The self-weighting design of the newest NIS version (2013 and later) delivers more stable and precise estimates than previous versions (2012 and earlier).

\section{Statistical analysis}

The patient demographics, occlusion site, and stratification based on time and imaging are descriptively presented and compared. All data analysis was performed using JMP statistical software, V.11 (SAS Institute Inc, Cary, North Carolina, USA). The significance of simple bivariate associations was assessed using Fisher's exact test for categorical variables, Student's t-test for continuous variables, or logistic regression, as appropriate.

\section{RESULTS}

\section{Rate of LVO among AIS discharges from all geographic} locations

A total of 2739 patients with AIS were discharged from hospital over the 3-year period. Of the 2691 patients for whom ethnicity information was available, the majority were white/Caucasian $(\mathrm{n}=2555,94.9 \%)$. There were $1157(42.2 \%)$ patients from the PSA, 798 (29.1\%) from the SSA, and 784 (28.6\%) patients from the rest of the state, neighboring counties from the surrounding states, or patients traveling through from more distant states. The mean age was $67.3( \pm 15.6)$ years and $1420(51.8 \%)$ were female. An LVO was present in $324(11.8 \%$, 95\% CI $10.7 \%$ to $13.1 \%)$ patients of whom $232(71.6 \%$, 95\% CI $66.5 \%$ to $77.2 \%)$ had an M1, $37(11.4 \%$, 95\% CI $8.4 \%$ to $15.3 \%)$ had an ICA-T and 55 (17\%, 95\% CI $13.3 \%$ to $21.4 \%)$ had a BA occlusion. The median National Institutes of Health Stroke Scale score was 17 (IQR 9-21) for M1 occlusion, 21 (IQR 15-25) for ICA-T occlusion, and 16 (IQR 4-26) for a BA occlusion $(\mathrm{p}=0.1)$. The majority of patients with LVO (174 $(53.7 \%, 95 \%$ CI $48.3 \%$ to $59.1 \%))$ presented within 6 hours of symptom onset. Forty-four $(13.6 \%$, 95\% CI $10.3 \%$ to $17.8 \%)$ patients presented within 6-12 hours, 87 (26.9\%, 95\% CI $22.3 \%$ to $31.9 \%$ ) after 12 hours, and 19 (5.9\%, 95\% CI $3.8 \%$ to $9 \%$ ) patients presented with an unknown time of symptom onset. Of the 269 patients with an anterior circulation LVO, $150(55.8 \%, 95 \%$ CI $49.8 \%$ to $61.2 \%)$ presented within 6 hours of symptom onset and 184 (68.4\%, 95\% CI 62.6 to 73.7) patients presented with an ASPECTS $\geq 6$. Among the 184 patients with favorable ASPECTS $\geq 6,121(65.8 \%, 95 \% \mathrm{CI}$ $58.6 \%$ to $72.2 \%)$ presented within 6 hours of symptom onset and $63(34.2 \%, 95 \%$ CI $27.8 \%$ to $41.4 \%)$ after 6 hours $(\mathrm{p}<0.0001, \mathrm{OR}=0.27,95 \%$ CI 0.16 to 0.46$)$.

There was no difference in the rate of LVO based on the geographic location: an LVO was confirmed in $129(11.1 \%)$ patients from the PSA, 97 (12.2\%) from the SSA, and 98 $(12.5 \%)$ patients from other regions $(p=0.12)$. A comparison of the presenting time from symptom onset and ASPECTS based on region (table 1) demonstrated that more patients from the PSA, SSA, or immediately adjacent counties of Pennsylvania presented within 6 hours symptom onset and had favorable ASPECTS than those presenting from more distant regions. 
Table 1 A comparison of the time from symptom onset and Alberta Stroke Program Early CT Score (ASPECTS) for patients admitted from different geographic areas served by the hospital

\begin{tabular}{|c|c|c|c|c|c|c|c|}
\hline & \multicolumn{2}{|c|}{ Time from onset (hours) } & \multirow[b]{2}{*}{ p Value } & & \multicolumn{2}{|c|}{ ASPECTS (anterior circulation) } & \multirow[b]{2}{*}{ p Value } \\
\hline & $<6$ n (\%) & $\geq 6 \mathrm{n}(\%)$ & & & $<6$ n (\%) & $\geq 6$ n (\%) & \\
\hline PSA $(n=129)$ & $75(58.1)$ & $54(41.9)$ & 0.03 & PSA $(n=109)$ & $29(26.6)$ & $80(73.4)$ & 0.07 \\
\hline SSA $(n=97)$ & $54(55.7)$ & $43(44.3)$ & & SSA $(n=82)$ & $26(31.7)$ & $56(68.3)$ & \\
\hline PA $(n=40)$ & $24(60)$ & $16(40)$ & & $P A(n=33)$ & $12(36.4)$ & $21(63.6)$ & \\
\hline Others* $(n=58)$ & $21(36.2)$ & $37(63.8)$ & & Others $(n=45)$ & $22(48.9)$ & $23(51.1)$ & \\
\hline
\end{tabular}

*Others: patients from more distant parts beyond the primary or the secondary service areas either from within the state or from neighboring states; PA: neighboring counties (Fayette, Greene) from PA (within 40 miles).

PA, Pennsylvania; PSA, primary service area (within 35-40 miles); SSA, secondary service area (greater than 40 miles).

\section{Incidence of LVOs based on the PSA}

Of the 1157 patients with AIS discharged from the PSA over the 3 years, 129 had an LVO (11.1\%, 95\% CI 9.5\% to $13.1 \%)$. The distribution of clot location was M1 in 102 patients $(79.1 \%, 95 \%$ CI $71.3 \%$ to $85.2 \%)$, ICA-T in seven patients $(5.4 \%, 95 \%$ CI $2.7 \%$ to $10.8 \%)$, and BA in 20 patients $(15.5 \%$, $95 \%$ CI $10.3 \%$ to $22.7 \%)$. Based on time, 75 patients $(58.1 \%$, $95 \%$ CI $49.5 \%$ to $66.3 \%)$ presented within 6 hours and 54 patients $(41.9 \%, 95 \%$ CI $33.7 \%$ to $50.5 \%)$ presented after 6 hours of symptom onset. Of the 109 patients with an anterior circulation occlusion, $80(73.4 \%$, 95\% CI $64.4 \%$ to $80.8 \%)$ presented with an ASPECTS $\geq 6$ and 29 (26.6\%, 95\% CI 19.2\% to $35.6 \%$ ) with an ASPECTS $<6$.

As mentioned all counties in the service areas report their annual discharges to the West Virginia Healthcare Authority. Thus based on the PSA population of 210000 , from the hospital's $85 \%$ share of patients with stroke from the PSA and by accounting for the remaining $15 \%$, we estimated an incidence of 24 (95\% CI 20 to 28) LVOs per 100000 people per year. A breakup of the rate per 100000 person-years based on time, clot location and ASPECTS is presented in table 2 together with an estimation of total numbers in each category for the US population for 2015. The results yield an estimated 695712 total cases of AIS for the USA, of which potentially 77569 (95\% CI 65835 to 91091 ) are LVOs (ICA-T, M1, BA). We estimated that 10284 mechanical thrombectomy procedures were performed in 2015 based on the previously described methodology.

\section{DISCUSSION}

This analysis yields an annual incidence of 24 LVOs (ICA-T, M1, BA) per 100000 people (95\% CI 9.5 to 13.1) based on the study's population, which is within a geographic region that has one of the highest stroke rates in the country. ${ }^{1}$ The extrapolated national incidence of 77569 (95\% CI 65835 to 91091 ) new LVOs per year thus probably represents the upper bounds of annual large vessel stroke estimates in the country. Since the total AIS estimate of 695712 patients in this study (table 2) is close to the 691650 estimate of patients with AIS generated from previous statistics, ${ }^{1}$ the estimated annual LVO burden is likely to be realistic. The $11 \%$ rate of LVO strokes in this cohort was based on all hospital discharges for AIS using the same ICD -9 codes that are typically used to determine the rate of ischemic stroke admissions-that is, ICD-9 433, 434, and 435. One previous study ${ }^{6}$ determined a higher rate of LVO among patients admitted with suspected stroke. However, since the estimates were based on clinically suspected strokes at admission and not all AIS discharges based on ICD-9 codes, the denominator might be smaller. That study also did not include a population analysis. The $11 \%$ rate in this study is based on the primary service population of 210000 people spread over 1492 square miles and living within 40 miles of the tertiary referral hospital. This may be a useful number for hospitals of similar size and rural geography to gauge their potential LVO population; however, application of the results to smaller or urban hospitals may not be accurate owing to different referral pathways.

An population eligible for endovascular treatment for anterior circulation LVOs based on the recent randomized clinical trials ${ }^{2-5}$ criteria of early presentation from symptom onset ( $<6$ hours) and favorable imaging (ASPECTS $\geq 6$ ) yielded potentially 10 treatment eligible LVO cases per 100000 person-years (95\% CI 8 to 11). However, in the study population there were an additional five patients per 100000 (95\% CI 3 to 6) with an ASPECTS $\geq 6$ presenting after 6 hours who might benefit from an intervention. Five patients per 100000 had a BA occlusion that could also be treated but their eligibility based on imaging was not assessed and so the true number is likely to be lower since some would present with completed infarcts incompatible with survival or a good outcome. Our assessment of these potential patients is very similar to a recent publication from Australia that estimated potential endovascular thrombectomy to range from 11 (95\% CI 4 to 18 ) to 22 (95\% CI 13 to 31) patients per 100000 person-years based on the restrictiveness of the inclusion criteria. ${ }^{7}$

True treatment eligibility could be lower than these estimates because patients with significant comorbidities, poor baseline functional status, or unfavorable vascular anatomy are not accounted for and can lower the target population. On the other hand, inclusion of patients with dominant M2 division occlusion can increase the target population. Additionally, measures that improve early detection of large vessel strokes, such as mobile CT scanners or portable devices that detect cerebral blood flow (or lack of it), may allow for early diversion of patients to a comprehensive treatment center. Similarly, consistently efficient interfacility transfer mechanisms may enable patients to reach a hospital delivering endovascular care earlier. Such division of labor among hospital systems can allow for appropriate and targeted allocation of resources. Within the comprehensive hospital, reducing door-to-needle times by following protocol-driven processes ${ }^{8}$ may further increase treatment effectiveness. We had a higher percentage of patients from the closer proximity PSA presenting within 6 hours of symptom onset with favorable imaging than patients coming from further away. Other studies have also shown a decay in ASPECTS during patient interfacility transfer. ${ }^{9}$ The hospital system in this study is constantly developing resources and capacity to stay ahead of the projected demand based on its referral network. In the future, elements of neuroprotection ${ }^{10}{ }^{11}$ and targeted 


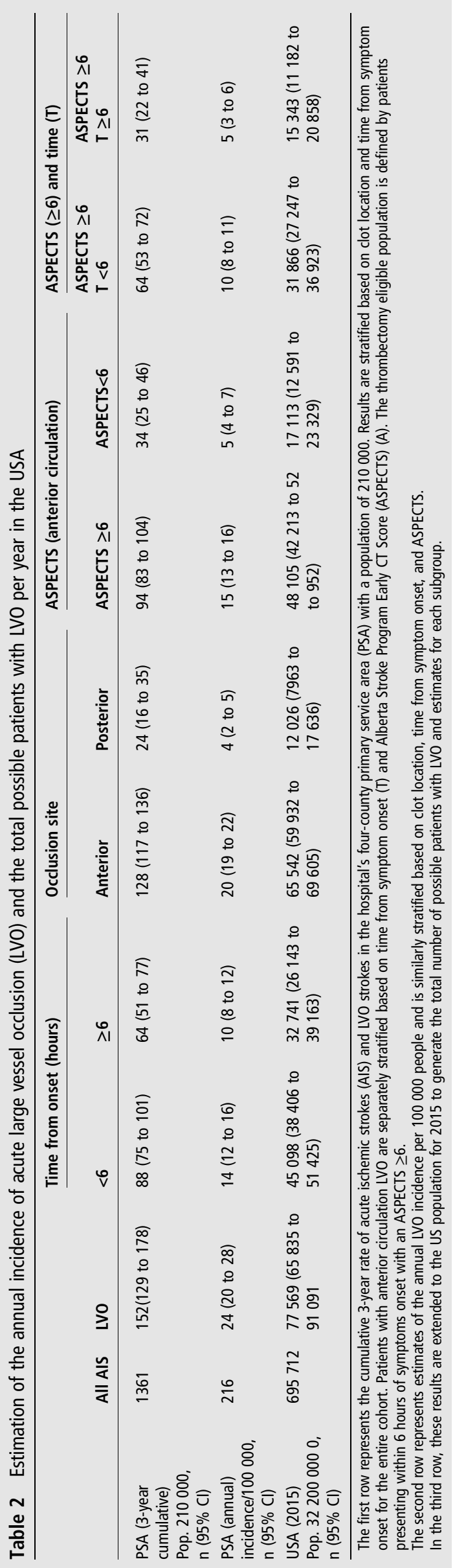

cerebral cooling ${ }^{12}{ }^{13}$ may be able to arrest or retard the core infarct progression before or after revascularization.

The study yielded an estimated 10284 mechanical thrombectomy procedures for 2015. This is based on Medicare data and private payer estimates generated from MedPAR and HCUP that include the NIS. Medicare data are fairly accurate because they are obtained from patient files submitted to Medicare by the hospitals. This is similar to an estimate based on informal sampling of busy stroke centers and their catchment population of about 10000 annual procedures. ${ }^{14}$ Nevertheless, this probably represents an underestimation of the real procedure volume as it does not account for any coding errors. Industry projections could be higher based on device sales and proprietary third party appraisals but might also overestimate the market based on inflated assumptions. Our estimate of 10284 mechanical thrombectomy procedures would translate into three thrombectomy procedures per 100000 people for 2015. A hypothetical estimate of 15000 and 20000 thrombectomy procedures for 2015 is equivalent to 4.6 and 6 procedures per 100000 people, respectively. A range of 3-6 annual thrombectomy procedures per 100000 person-years is still less than the potential stroke interventions that can be performed just for the 'thrombectomy eligible' population proved to benefit from endovascular therapy. By eliminating time from patient selection and focusing only on favorable imaging there could be as many as 15 thrombectomy eligible patients per 100000 person-years in those with an anterior circulation occlusion alone. Including treatment eligible posterior circulation strokes and M2 occlusions further increases this number. Thus a current annual rate of 3-6 thrombectomy procedures per 100000 people suggests a twoto fivefold increase in the potential volume of endovascular stroke interventions.

Patients with large vessel stroke are thus currently underserved. This is not necessarily due to the lack of a trained physician workforce ${ }^{15-17}$ but perhaps more due to prehospital and intrahospital systems of care that need further development. The strong evidence in favor of intervening in patients with LVOs will probably enhance these care pathways, increase patient awareness, and increase acceptance of these procedures as the standard of care. An initial goal could be to reach 10 stroke interventions per 100000 people per year to at least treat those patients who have been shown to benefit the most. Some centers could be meeting or exceeding this target for their catchment population now, whereas others could use this goal as a marker to measure progress in disseminating this care. Perhaps parallel progress in emergency medical services, early diagnosis and neuroprotection could accelerate these next steps in evolution of stroke care.

One important point to keep in mind as this care evolves is the realistic and perhaps cautionary trend observed in similar stages of percutaneous coronary intervention (PCI) development. In the Global Registry of Acute Coronary Events (GRACE) about $30 \%$ of patients with acute coronary syndrome admitted within 12 hours of symptom onset underwent PCIs. ${ }^{18}$ Other studies have reported a rate of $46-75 \%$ rate of coronary interventions for acute ST-segment elevation myocardial infarction (STEMI). ${ }^{18-21}$ The GRACE registry also showed that between 1999 and 2006, primary PCIs for patients admitted with STEMI grew at about 30\% for the first 2 years, $19 \%$ for the next 2 years, and dropped to an average of $9 \%$ for the last 2 years ${ }^{22}$ and has declined further since then. ${ }^{23}{ }^{24}$ It is also important to note that even the steepest upslope of the acute coronary intervention era did not reach an annual 30\% growth rate and that too only for the initial years. This is despite 
significant efforts by the American Heart Association to develop systems of care, raise public awareness, and set treatment guidelines. Thus it is prudent to recognize that we will never treat every eligible patient with LVO and account for the inherent limitations within any healthcare system when projecting volumes and developing infrastructure.

\section{Limitations}

The 11\% LVO rate among all AIS admissions represents data from a large, academic tertiary-level hospital in a rural geographic area. This rate may be different for smaller hospitals or those in an urban environment. Thus universal application of this LVO rate could be misleading unless other variables are taken into account. The general incidence and prevalence of AIS also varies from region to region and the LVO rate estimated in our primary population may be different from that in another. However, based on the above average stroke incidence in our state, the calculated LVO incidence based on our population probably constitutes the upper limits of these estimates.

Treatment eligibility based on time from symptom onset can also vary according to regional resources and patient transfer mechanisms. A rural area such as ours with one large tertiary center receiving the bulk of patients with AIS may have a different stratification of patients than centers in large urban environments or centers receiving a large amount of transfers from more distant smaller hospitals.

A limitation of our methodology in estimating numbers of mechanical thrombectomy procedures is that the HCUP data we used are from 2013 since the 2014 data are not available until the fall of 2016 according to the NIS. HCUP data always lag behind Medicare data and therefore our calculated numbers probably underestimate the true procedure volume. For this reason, in our discussion we include a range up to twice the volume of our estimation to compare the LVO incidence with treated patients and to assess the potential growth in thrombectomy procedures.

\section{CONCLUSIONS}

The incidence of acute LVO confirmed by imaging was estimated to be 24 per 100000 person-years (95\% CI 20 to 28), which is in keeping with a similar recent publication. ${ }^{7}$ This translates into 77569 (95\% CI 65835 to 91091$)$ potential new patients with LVO per year in the USA, based on the 2015 population. This estimate may be reasonable as the total cases of AIS estimated in this population-based study of 695712 is very similar to the 691650 AIS estimate from the American Heart Association publication. ${ }^{1}$ Thrombectomy eligible patients with an anterior circulation occlusion were estimated at 10 per 100000 person-years with an additional treatment eligible five patients per 100000 person-years presenting with favorable imaging after 6 hours of symptom onset and four patients per 100000 person-years presenting with BA occlusion. This number does not include potentially treatable M2 occlusions. Thus the total number of thrombectomy eligible patients could range from 10 to 20 patients per 100000 person-years. An estimated range of 10000-20000 annual thrombectomy procedures yields 3-6 mechanical thrombectomies per 100000 person-years, indicating a significant potential for growth. The history of coronary interventions illustrates that it will be impossible to treat every eligible patient despite all possible efforts; however, developing systems of care can steadily increase the proportion of eligible patients who are treated and these estimates can serve as markers for gauging progress.
Contributors ATR: study design, data analysis, manuscript preparation. AES, SHB, PSL, JRD, ART, NL-W: data collection. JSC: manuscript review, data collection.

Competing interests ATR has a consulting agreement with Stryker Neurovascular. Ethics approval Institutional Review Board.

Provenance and peer review Not commissioned; externally peer reviewed.

Open Access This is an Open Access article distributed in accordance with the Creative Commons Attribution Non Commercial (CC BY-NC 4.0) license, which permits others to distribute, remix, adapt, build upon this work non-commercially, and license their derivative works on different terms, provided the original work is properly cited and the use is non-commercial. See: http://creativecommons.org/ licenses/by-nc/4.0/

\section{REFERENCES}

1 Mozaffarian D, Benjamin EJ, Go AS, et al. Heart disease and stroke statistics2015 update: a report from the American Heart Association. Circulation 2015;131: e29-322.

2 Berkhemer OA, Fransen PS, Beumer D, et al. A randomized trial of intraarterial treatment for acute ischemic stroke. N Engl J Med 2015;372:11-20.

3 Campbell BC, Mitchell PJ, Kleinig TJ, et al. Endovascular therapy for ischemic stroke with perfusion-imaging selection. N Engl J Med 2015;372:1009-18.

4 Saver JL, Goyal M, Bonafe A, et al. Stent-retriever thrombectomy after intravenous t-PA vs. t-PA alone in stroke. N Engl J Med 2015;372:2285-95.

5 Goyal M, Demchuk AM, Menon BK, et al. Randomized assessment of rapid endovascular treatment of ischemic stroke. N Engl J Med 2015;372:1019-30.

6 González RG, Furie KL, Goldmacher GV, et al. Good outcome rate of $35 \%$ in IV-tPA-treated patients with computed tomography angiography confirmed severe anterior circulation occlusive stroke. Stroke 2013;44:3109-13.

7 Chia NH, Leyden JM, Newbury J, et al. Determining the number of ischemic strokes potentially eligible for endovascular thrombectomy: a population-based study. Stroke 2016;47:1377-80.

8 Rai AT, Smith MS, Boo S, et al. The 'pit-crew' model for improving door-to-needle times in endovascular stroke therapy: a Six-Sigma project. J Neurointerv Surg 2016;8:447-52

9 Sun $\mathrm{CH}$, Connelly $\mathrm{K}$, Nogueira RG, et al. ASPECTS decay during inter-facility transfer predicts patient outcomes in endovascular reperfusion for ischemic stroke: a unique assessment of dynamic physiologic change over time. J Neurointerv Surg 2015;7:22-6.

10 Maniskas ME, Roberts JM, Aron I, et al. Stroke neuroprotection revisited: intra-arterial verapamil is profoundly neuroprotective in experimental acute ischemic stroke. J Cereb Blood Flow Metab 2016;36:721-30.

11 Lioutas VA, Novak V. Intranasal insulin neuroprotection in ischemic stroke. Neural Regen Res 2016;11:400-1.

12 Horn CM, Sun CH, Nogueira RG, et al. Endovascular reperfusion and cooling in cerebral acute ischemia (ReCCLAIM I). J Neurointerv Surg 2014;6:91-5.

13 Hwang $Y$, Jeon JS, Kim YW, et al. Impact of immediate post-reperfusion cooling on outcome in patients with acute stroke and substantial ischemic changes. J Neurointerv Surg Published Online First: 3 Mar 2016. doi:10.1136/neurintsurg2015-012233

14 Rai AT. Red pill, blue pill: reflections on the emerging large vessel stroke 'market'. J Neurointerv Surg 2015;7:623-5.

15 Jabbour P, Fiorella D. Are we training too many neuroendovascular fellows? World Neurosurg 2013;79:9-10.

16 Zaidat 00, Lazzaro M, McGinley E, et al. Demand-supply of neurointerventionalists for endovascular ischemic stroke therapy. Neurology 2012;79(Suppl 1):S35-41.

17 Rai AT. The stroke interventionalist. J Neurointerv Surg 2016;8:333-4.

18 Goodman SG, Huang W, Yan AT, et al. The expanded Global Registry of Acute Coronary Events: baseline characteristics, management practices, and hospital outcomes of patients with acute coronary syndromes. Am Heart J 2009;158:193-201.e1-5.

19 McManus DD, Gore J, Yarzebski J, et al. Recent trends in the incidence, treatment, and outcomes of patients with STEMI and NSTEMI. Am J Med 2011;124:40-7.

20 Ryan J, Linde-Zwirble W, Engelhart L, et al. Temporal changes in coronary revascularization procedures, outcomes, and costs in the bare-metal stent and drug-eluting stent eras: results from the US Medicare program. Circulation 2009;119:952-61.

21 Gerber Y, Rihal CS, Sundt TM III, et al. Coronary revascularization in the community. A population-based study, 1990 to 2004. J Am Coll Cardiol 2007;50:1223-9.

22 Eagle KA, Nallamothu BK, Mehta RH, et al. Trends in acute reperfusion therapy for ST-segment elevation myocardial infarction from 1999 to 2006: we are getting better but we have got a long way to go. Eur Heart J 2008:29:609-17.

23 Bradley SM, Bohn CM, Malenka DJ, et al. Temporal trends in percutaneous coronary intervention appropriateness: insights from the clinical outcomes assessment program. Circulation 2015;132:20-6.

24 Riley RF, Don CW, Powell W, et al. Trends in coronary revascularization in the United States from 2001 to 2009: recent declines in percutaneous coronary intervention volumes. Circ Cardiovasc Qual Outcomes 2011;4:193-7. 